\title{
$\pi \pi$ AMPLITUDES FITTED TO EXPERIMENTAL DATA AND TO ROY'S EQUATIONS
}

\author{
R. KAMIŃSKI, L. LEŚNIAK \\ Henryk Niewodniczański Institute of Nuclear Physics, Polish Academy of Sciences, \\ ul. Radzikowskiego 152, PL 31-342, Kraków, Poland \\ B. LOISEAU \\ LPNHE, Université P. et M. Curie, 4, Place Jussieu, \\ 75252 Paris Cedex 05, France
}

\begin{abstract}
The scalar-isoscalar, scalar-isotensor and vector-isovector $\pi \pi$ amplitudes are fitted simultaneously to experimental data and to Roy's equations. The resulting amplitudes are compared with those fitted only to experimental data. No additional constraints for the $\pi \pi$ threshold behaviour of the amplitudes are imposed. Threshold parameters are calculated for the amplitudes in the three waves. Spectrum of scalar mesons below $1.8 \mathrm{GeV}$ is found from the analysis of the analytical structure of the fitted amplitudes.
\end{abstract}

Keywords: pion-pion interactions; Roy's equations; threshold parameters.

The $\pi \pi$ amplitudes in the scalar-isoscalar $(S 0)$, the scalar-isotensor $(S 2)$ and the vector-isovector $(P 1)$ waves have already been analyzed by us with the help of Roy's equations 11. Our aim was to eliminate the "up-down" ambiguity in the $S 0$ phase shifts below $1 \mathrm{GeV} 2$. We have shown that only amplitudes reproducing the "downflat" data set fulfill Roy's equations. Therefore they satisfy crossing symmetry and should then correspond to physical amplitudes.

The aim of this talk is to present preliminary results in a construction of the theoretical $\pi \pi$ amplitudes which simultaneously describe the experimental data and fulfill Roy's equations up to $1150 \mathrm{MeV}$. In order to study the constraints from Roy's equations we also calculate amplitudes fitted only to experimental data in the three channels ( fit 1). The analytical form of the $S 0$ wave has been obtained in the coupled three channel model 3 . Now a new fit to the "down-flat" data from the $\pi \pi$ threshold up to $1600 \mathrm{MeV}$ is performed. In the $S 2$ wave we have used the same model as in 1 and the data corresponding to the solution A of Hoogland et al. 4. In the $P 1$ wave we have introduced a two channel model with a rank-two and a rank-one separable potentials in the $\pi \pi$ and $K \bar{K}$ channels, respectively. Amplitude of the $P 1$ wave has

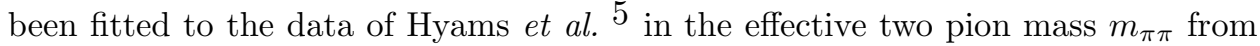
600 up to $1400 \mathrm{MeV}$. The numbers of free parameters in the $S 0, S 2$ and $P 1$ waves are 14,4 and 8 , respectively.

*Unité de Recherche des Universités Paris 6 et Paris 7, associée au CNRS. 
The total $\chi^{2}$ used in the simultaneous fit to experimental data and to Roy's equations (fit 2) has been defined in our previous work 1. It consists of two parts: $\chi_{\text {exp }}^{2}$ - related to the experimental data and $\chi_{\text {Roy }}^{2}$ - related to Roy's equations. The latter one is defined as the sum of three terms for the isospin $I=0,1,2$ in the $s=m_{\pi \pi}^{2} / m_{\pi}^{2}$ range from 4.1 up to 67 . The 23 points in the calculations of $\chi_{R o y}^{2}$ have been chosen as follows: $s_{1}=4.1, s_{2}=5$ and $s_{j}=3 j-2$ for $j=3,4 \ldots 23$.

In Table 1 the $\chi^{2}$ components for the experimental data and for Roy's equations are presented. It can be seen that while the data for the phase shifts $\delta_{J=0}^{I=0}$ and inelasticity $\eta_{0}^{0}$ in the $S 0$ wave are similarly well described in both fits, the data for $S 2, P 1$ and the data $\underline{6}$ for the sum $\delta_{\pi \pi}+\delta_{K \bar{K}}$ are better reproduced in fit 1 . One can conclude that some parts of these data do not satisfy the constraints imposed by crossing symmetry.

The real parts of the amplitudes from fit 1 and fit 2 are shown in Fig. 1. Again one can see that the agreement of the experimental data in the $S 2$ and $P 1$ waves with the amplitudes of the fit 2 is not as good as that of the fit 1 . In the fit 2 the averaged values of differences between real parts of amplitudes used as input in Roy's equations and real parts of amplitudes obtained as output are smaller than $(2,1$ and 3$) \times 10^{-3}$ for the $S 0, S 2$ and $P 1$ waves, respectively.

Table 1. Values of various components of $\chi_{\text {tot }}^{2}$ with the corresponding numbers of data points in parentheses. References to experimental data can be found in ${ }^{1}$. Symbol $\phi$ denotes sum $\delta_{\pi \pi}+\delta_{K \bar{K}}$.

$\begin{array}{ccccccccccccc}\text { Fit } & \chi_{\text {tot }}^{2} & \chi_{\text {Royo }}^{2} & \chi_{\text {Roy }}^{2} & \chi_{\text {Roy }}^{2} & \chi_{\text {Roy }}^{2} & \chi_{\delta_{0}^{0}}^{2} & \chi_{\eta_{0}^{0}}^{2} & \chi_{\phi}^{2} & \chi_{\delta_{0}^{2}}^{2} & \chi_{\delta_{1}^{1}}^{2} & \chi_{\eta_{1}^{1}}^{2} & \chi_{\exp }^{2} \\ & (248) & (23) & (23) & (23) & (66) & (56) & (30) & (21) & (12) & (40) & (20) & (179) \\ 1 & 123.5 & - & - & - & - & 48.7 & 28.7 & 10.7 & 1.9 & 20.1 & 13.4 & 123.5 \\ 2 & 247.5 & 7.1 & 2.7 & 16.0 & 25.8 & 59.7 & 30.3 & 33.9 & 26.2 & 49.0 & 22.6 & 221.7\end{array}$

In Table 2 values of the scattering lengths $a_{J}^{I}$ and the slope parameters $b_{J}^{I}$ as defined in our paper 1 and positions of the most important $S$-matrix poles $\frac{3}{1}$ for the $S 0$ wave are presented. In the fit 1 the threshold parameters of the $S 2$ and $P 1$ amplitudes are much different from those calculated for the fit 2. In Fig. 1 one can see significant differences in the $\pi \pi$ threshold behaviour of the $S 2$ and $P 1$ amplitudes. This is due to a lack of experimental data near the $\pi \pi$ threshold in these waves and a lack of constraints from Roy's equations in the fit 1 . Note also the differences in the positions of the poles. Positions of the $\rho$ meson poles depend also on the fit. They have been calculated for $\operatorname{Im} k_{\pi}<0, \operatorname{Im} k_{K}>0$ and are $(764-i 55) \mathrm{MeV}$ and $(765-i 72) \mathrm{MeV}$ for the fit 1 and fit 2 , respectively.

Table 2. Values of $a_{J}^{I}, b_{J}^{I}$ and pole positions for the $S 0$ wave in the complex energy plane (in MeV) on sheets denoted by signs of imaginary parts of the momenta in three channels

$\begin{array}{cccccccccc}\text { Fit } & a_{0}^{0} & b_{0}^{0} & a_{0}^{2} & b_{0}^{2} & a_{1}^{1} & b_{1}^{1} & (-,+,+) & (-,+,+) & (-,-,-) \\ 1 & 0.210 & 0.255 & -0.097 & -0.064 & 0.32 & -0.017 & 475-i 242 & 1008-i 32 & 1415-i 85 \\ 2 & 0.204 & 0.243 & -0.044 & -0.076 & 0.047 & 0.0072 & 450-i 280 & 981-i 50 & 1474-i 73\end{array}$

Work to consider, in our $\pi \pi$ amplitudes, QCD low energy constraints from Chiral Perturbation Theory is in progress. 

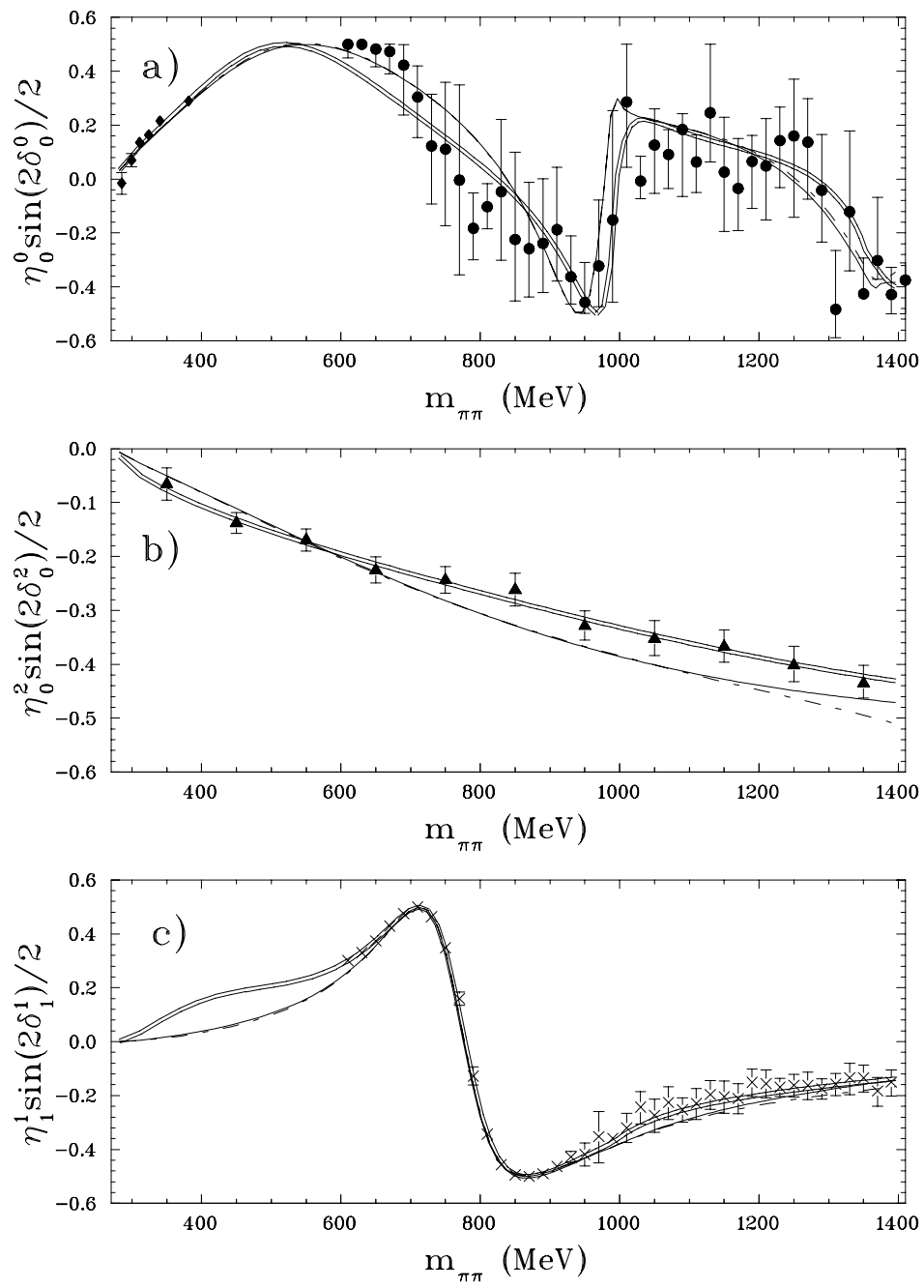

Fig. 1. Real parts of the $\pi \pi$ amplitudes multiplied by $2 k / m_{\pi \pi}$ ( $k$ is the relative momentum in the $\pi \pi$ system). Double solid lines correspond to fit 1 . Single solid and dashed lines correspond to the "input" and the "output" amplitudes, respectively for the fit 2. References to experimental data can be found in ${ }^{1}$.

\section{References}

1. R. Kamiński, L. Leśniak, B. Loiseau Phys. Lett. B551, 241 (2003).

2. R. Kamiński, L. Leśniak, K. Rybicki, Z. Phys. C74, 79 (1997).

3. R. Kamiński, L. Leśniak, B. Loiseau, Phys. Lett. B413, 13 (1997); Eur. Phys. J. C9, 141 (1999).

4. W. Hoogland et al., Nucl. Phys. B126, 109 (1977).

5. B. Hyams et al., Nucl. Phys. B64, 134 (1973).

6. D. Cohen et al., Phys. Rev. D22, 2595 (1980). 